\title{
Integrating Hydrodynamic and Hydraulic Modeling for Evaluating Future Flood Mitigation in Urban Environments
}

\author{
Mahesh Prakash ${ }^{1, *}, J^{2}$ ames Hilton ${ }^{1}$, and Lalitha Ramachandran ${ }^{2}$ \\ ${ }^{1}$ CSIRO Digital Productivity Flagship, Melbourne, Australia \\ ${ }^{2}$ The City of Port Phillip, VIC, Australia \\ Mahesh.Prakash@csiro.au
}

\begin{abstract}
We present an integrated flood modelling tool that is able to evaluate different mitigation solutions for areas that are prone to floods from storm surge and heavy rainfall. Our model integrates catchment and coastal flood modelling (spatio-temporally dynamic), including sea level rise, to provide a holistic inundation model for future flooding. Additionally, the model aims to enable simulation of a combination of flood mitigation and adaptation options. To date, the practice has been to model either drainage augmentation solutions alone, or (for coastal inundation) single coastal adaptation solutions. This tool aims to deliver the capacity to model a range of both coastal and drainage adaptation solutions to understand what combination of solutions might be effective. The model is demonstrated for example cases in the City of Port Phillip, Melbourne. Three mitigation strategies involving the use of a hypothetical off-shore reef and the combination of a single valve systems and retention/detention measures are evaluated for the region around Elwood canal for current and future scenarios.
\end{abstract}

Keywords: modular mitigation analysis, drainage augmentation, local councils, evidence based cost-benefit analysis, land-use planning.

\section{Introduction}

Australia has a very long coastline stretching almost $36,000 \mathrm{~km}$ in the mainland. A significant percentage of the population resides along this coastline especially along the eastern seaboard. Among these urban environments there are several locations that are low lying and are prone to flooding including storm surge, heavy rainfall and catchment. The Elwood Canal region in the City of Port Phillip (CoPP) in Melbourne, Victoria is particularly at risk of flooding which has been indicated through flood mapping studies carried out internally by engineers in the council as well as through AECOM [1]. However the coastal flood inundation studies have been carried out using essentially static bath tub type models which do not provide details of flooding such as duration and areas that are prone to water logging (lack of sufficient drainage). This can be particularly important to decide future infrastructure needs including the effect of sea level rise (SLR). Additionally mitigation and adaptation

\footnotetext{
* Corresponding author.
} 
options have not been investigated using a simulation and modelling approach in the past following on from a flood modelling exercise. An integrated flood modelling tool that is able to evaluate different mitigation solutions has therefore been developed by CSIRO for the CoPP so that the council:

1. Continues to have new (as becomes available) catchment and coastal data modelled into an integrated hydrodynamic model in order to understand what climate risks might present design and maintenance challenges to a range of municipal assets and land use planning processes. Specifically, we want to have a clear perspective on the future behaviour (shape, extent and duration) of flooding, and what changes how and when.

2. Is able to model a range of adaptation solutions and land use planning changes to test their effectiveness in mitigating flooding, in order to understand what future infrastructure and land-use needs might be.

These then give councils a basis for understanding where and when critical infrastructure and land-use changes may need to be made, as well as the required investment plan for new adaptive infrastructure. The tool is currently being investigated by the Association of Bayside Municipalities (ABM), Victoria for Port Phillip bay-wide deployment.

\section{Key Components of the Tool}

The mitigation modeling tool being developed consists of the following key components:

1. Hydrodynamic Module - A 2D shallow water based model will be used to simulate the flow hydrodynamics for the scenarios. The solver is able to concurrently simulate flood inundation due to sea level rise, storm surge, tides and rainfall allowing scenarios with multiple flood interactions possible. This is especially important for coastal councils where there is a significant likelihood of combined coastal and catchment flooding.

2. Topography Clean-Up Module (Terrain/Bathymetry) - The solver is integrated with a LiDAR pre-processing capability that allows input of raw/processed LiDAR data at varying resolutions to be used as input into the hydrodynamic model. The model allows specification of variable drag maps resulting from regions with varying vegetation composition and other resistance (infrastructure) overlays.

3. Hydraulic Module - Drainage is handled either as an infiltration sink or by using an explicit 1D network model connection into the 2D solver for complex urban drainage infrastructure.

4. Mitigation Module - Mitigation solutions are integrated into the hydrodynamic/hydraulic model either as modules (eg: drainage augmentation, retention/detention systems) or as structures that can be designed in AutoCAD or similar and imported into the model.

5. Analysis and Visualisation Module - An integrated visualisation tool allows the results of the hydrodynamic solver to be produced in a visual 3D Google Earth-like environment. The flood inundation can be visualised as depth/velocity/force maps to provide a before and after quantitative assessment of the assessed mitigation scenario(s). 
A detailed description of the computational models and the mitigation models is provided in the sections below.

\section{Computational Models}

The tidal and pluvial hydrodynamics were modelled using a shallow water model developed by CSIRO. The method has been specially adapted for modelling widespread areas of urban inundation. The model is well suited to both heavy rainfall events and tidal inundation. Recent applications include the impact of heavy rainfall events leading to the flash floods in Toowoomba in 2012 and the effects of possible SLR in the Kakadu region. The shallow water equations represent the depth averaged height and unit discharge of water above a fixed datum. The equations are discretised onto a fixed grid representing the land surface and computationally processed to predict the water dynamics. The underlying method is based on the finite-volume methods presented by Kurganov and Petrova [2]. The method requires a combined topological and bathymetric height map above a given vertical datum as well as an initial distribution of water. In addition the method allows the input of a given boundary condition, which may represent a storm surge or tsunami, as well as a spatially distributed source map, which may represent a rainfall or other water source. The effect of base friction is imposed using a Manning drag model.

The underground drains were incorporated into the hydrodynamic model using a 1D pipe network model (see Fig. 1). The solution to the 1D pipe network is derived as a steady state output at every hydrodynamic model time step. This integrated solution provides an accurate estimate of the dynamic flooding across a large urban region. The models have been extensively tested and validated against benchmark test cases presented by Neelz and Pender [3].

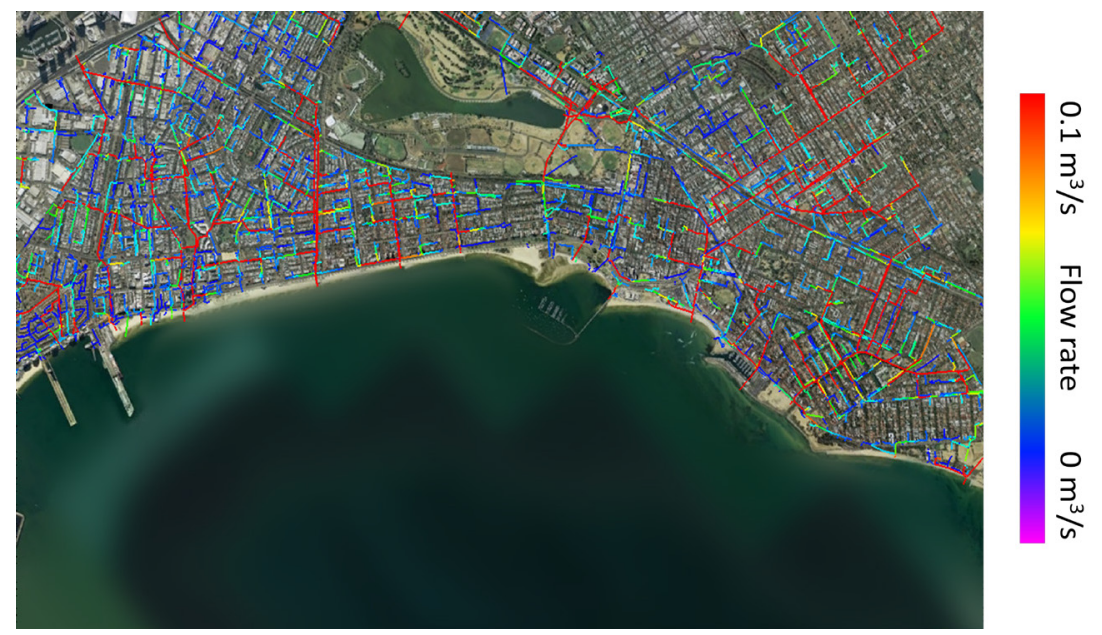

Fig. 1. Flow in drainage network for the City of Port Phillip 3 hours after a 1 in a 10 year ARI rainfall 


\section{Mitigation Models}

A highlight of the software tool is the ability to incorporate different types of mitigation options as applications which then can be deployed to specific parts of the region of interest. The mitigation models can be classified into three broad categories:

- Drainage augmentation: These can be either a simple measure such as increasing the size of the drainage pipe, deploying back valves into existing drainage pipes or enhancing drainage capacity by incorporating pumps at specified locations in the urban drainage system.

- Retention/detention systems: Retention/detention systems are typically useful for low to medium level flooding where a water storage system is also utilized for managed release of water to provide for additional capacity during a predicted flood event (such as a flash flood). Retention/detention systems can either be at a household level (tanks), street level (underground large storage tanks) and/or large parks/open spaces used as dug-out storage/release facilities.

- Sea walls/off-shore reefs/protective barriers: These are large engineering or natural (such as mangroves) protective barriers that are specifically designed to protect a region in a council for its economic, heritage and/or environmental value typically against storm surge or similar events.

The software tool is able to incorporate all three forms of mitigation models. The drainage augmentation and retention/detention systems are built in the tool in such a way that they can be modularly incorporated into any mitigation analysis either by a point and click mechanism or by assigning the mitigation model(s) to a particular chosen location in the council. The protective barriers are incorporated typically via an AutoCAD or similarly designed input. This is because protective barriers are specifically designed for a region of interest and cannot be modularized.

\section{Data Inputs}

The data requirements for the tool are summarized below:

- Terrestrial and bathymetric LiDAR data

- Land-use classification data to derive a variable drag map for the region of interest

- Historical storm surge data

- Tidal data

- Sea level rise scenario(s)

- Rainfall data

- Drainage network information (ArcGIS compatible)

- Mitigation solutions

- Modular (retention/detention, back valves, pumps)

- Designed (AutoCAD or similar for custom sea walls and protective barriers and off shore reefs)

- Historic flood data for validation

- Previously performed flood study information for calibration 


\section{Modelling Process Flow Chart}

The mitigation modeling process involves several components that have a specific purpose, these are described below, Fig. 2 provides a graphical flowchart summary describing the steps involved in the integrated mitigation modeling process:

1. Adoption of existing flood study data as input into an integrated flood mitigation model.

Purpose: Effective use of existing data and to reduce rework. Existing data will also be used for validation purposes.

2. Statistical extreme event analysis

Purpose: To understand and estimate the occurrence likelihood of extreme events such as storm surges and rainfalls for current day and for the future. This is important since there could be large variations in climatic conditions in a study region of interest. Rigorous statistical analysis provides a better basis for estimating flood frequency and amplitude. Using this approach will provide an envelope that can then be used as input into a hydrodynamic/hydraulic model for scenario simulations.

3. Statistical joint probability analysis

Purpose: To understand and estimate the occurrence likelihood of joint flood events such as coastal and catchment flooding for current day and for the future. Very few of the previous flood studies investigated the joint likelihood of flooding. Joint probability analysis is an emerging and robust approach for a holistic analysis of encompassing all possible avenues for flooding.

4. Integration of hydrodynamic modelling with a comprehensive drainage network (hydraulic) model

Purpose: To accurately predict flooding in an urban environment. A key outcome from the case study (described below) conducted for the City of Port Phillip study (2014) was that drainage networks contribute to flood inundation especially when there is a storm surge event and/or sea level rise due to the drainage pipes working as a reverse carrier of water from the sea further inland. This will be especially important for low-lying inland locations.

5. Incorporation of outputs from the statistical modelling into the hydrodynamic/hydraulic model.

Purpose: For simulation scenario analysis that uses an occurrence likelihood approach including joint (coastal and catchment) flood events. This provides a robust statistically based envelope for flood inundation predictions that is relevant for coastal councils.

6. Analysis of future flooding to investigate the effect of sea level rise.

Purpose: This is important since any future infrastructure (overland or underground) will need to be justified from a cost/benefit perspective.

7. Integration of suggested mitigation and adaptation strategies. 
Purpose: This relates to quantitatively assessing the effect of suggested mitigation and adaptation strategies for specific "hot spot" locations. This approach provides a quantitative method of assessing the effectiveness of flood mitigation and adaptation strategies especially for future flooding.

8. Provide further guidance on flood mitigation following on from the integrated analysis in step 7 above.

Purpose: This is a feedback loop that allows the planners/engineers to test various suggested strategies and potentially enhance their effectiveness.

The modelling components are linked and packaged (see Fig. 3) utilizing the CSIRO Workspace: A scientific workflow platform [4]. Workspace has the following advantages that are advantageous for the development of the mitigation modelling tool including:

1. Ability to modularly include different mitigation options

2. Ability to seamlessly connect computational models such as the $2 \mathrm{D}$ hydrodynamic model with the 1D network model for drainage

3. In-built capability to manage distributed computing such that a set of simulation scenarios can be assigned to different GPUs/CPUs and results drawn back into a unified analysis/visualization environment

4. In-built visualization facility

\section{$7 \quad$ Case Studies}

\subsection{Simulation Scenarios}

Table 1 summarises the simulation scenarios investigated for the integrated flood impact study. These scenarios were derived following on from a preliminary joint probability analysis as described in section 6 above. A maximum storm surge height of 1.3 $\mathrm{m}$ was chosen on the basis of the gauge measurements at St Kilda marina by Melbourne Water [5]. It is acknowledged that the maximum storm surge height recorded in St Kilda marina is higher than the one recorded at Williamstown $(1.15 \mathrm{~m})$ in the work of McInnes et al. [6]. The higher value is used for the purposes of evaluating a severe but very likely future scenario. The rainfall input condition was finalised following on from discussions with Melbourne Water for a typical storm event in CoPP. The same scenarios were repeated for evaluating the effect of mitigation described in section 4 .

Table 1. Simulation scenarios

\begin{tabular}{|c|c|c|c|c|c|}
\hline No & $\begin{array}{c}\text { Max. } \\
\text { Stm ht. }(\mathrm{m})\end{array}$ & $\begin{array}{c}\text { Rain } \\
(\text { ARI })\end{array}$ & $\begin{array}{c}\text { Rain } \\
(\mathrm{hrs})\end{array}$ & $\begin{array}{c}\text { SLR } \\
(\mathrm{m})\end{array}$ & $\begin{array}{c}\text { Max ht } \\
(\text { AHD })\end{array}$ \\
\hline 1 & 1.30 & 100 & 3 & 0.0 & 1.30 \\
\hline 2 & 1.30 & 100 & 3 & 0.4 & 1.70 \\
\hline 3 & 1.30 & 100 & 3 & 0.8 & 2.10 \\
\hline 4 & 1.30 & 100 & 3 & 1.1 & 2.40 \\
\hline
\end{tabular}




\subsection{Designed Mitigation - Off-Shore Reef}

The first case study is used to demonstrate the application of the tool to evaluate the effectiveness of a hypothetical off-shore reef designed to attenuate the effect of a storm surge wave impacting the Elwood region in the City of Port Phillip. The offshore reef was introduced into the model by importing a design concept proposed by Prof. Rob Roggema (Urban Design, Swinburne University, Melbourne). The offshore reef is $3 \mathrm{~m}$ tall, $150-200 \mathrm{~m}$ in width and approximately $2 \mathrm{~km}$ offshore. Fig. 4 shows the case without the seawall (left) and with the seawall (right), where the initial water level is shown in blue to highlight the difference between the maps. Fig. 5 shows the change in maximum flood extent without and with the off-shore reef for a high storm surge for current day conditions.

\subsection{Modular Mitigation - Back Calves and Retention/Detention}

In the second example two mitigation models (as shown in Fig. 6) have been investigated namely:

1. Non return valves included along the stretch of Elwood canal and main drains at St Kilda and end of Langridge Street and

2. Retention detention consisting of water tanks installed on all lots in the Elwood region, along roads identified as being prone to flooding and a cricket oval in north-west of Elsternwick Park.

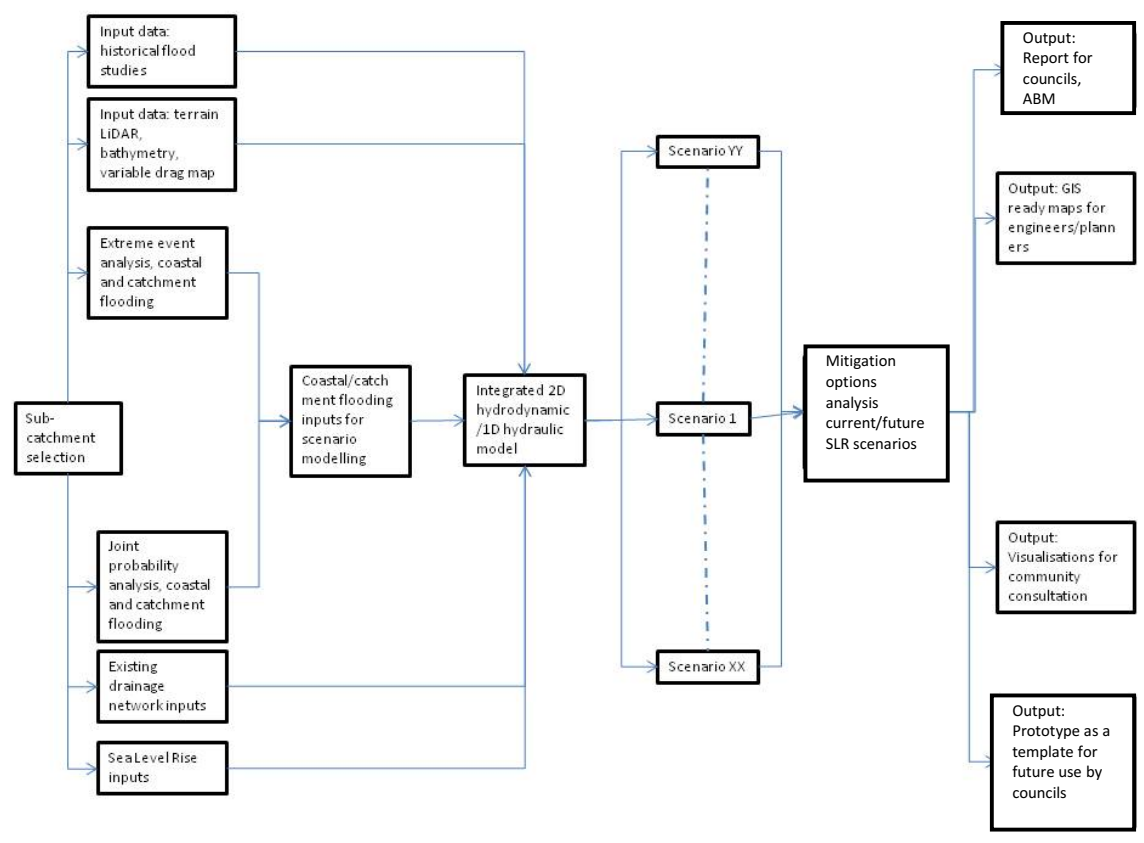

Fig. 2. Flowchart describing the steps involved in the integrated assessment of flood mitigation and adaptation strategies 


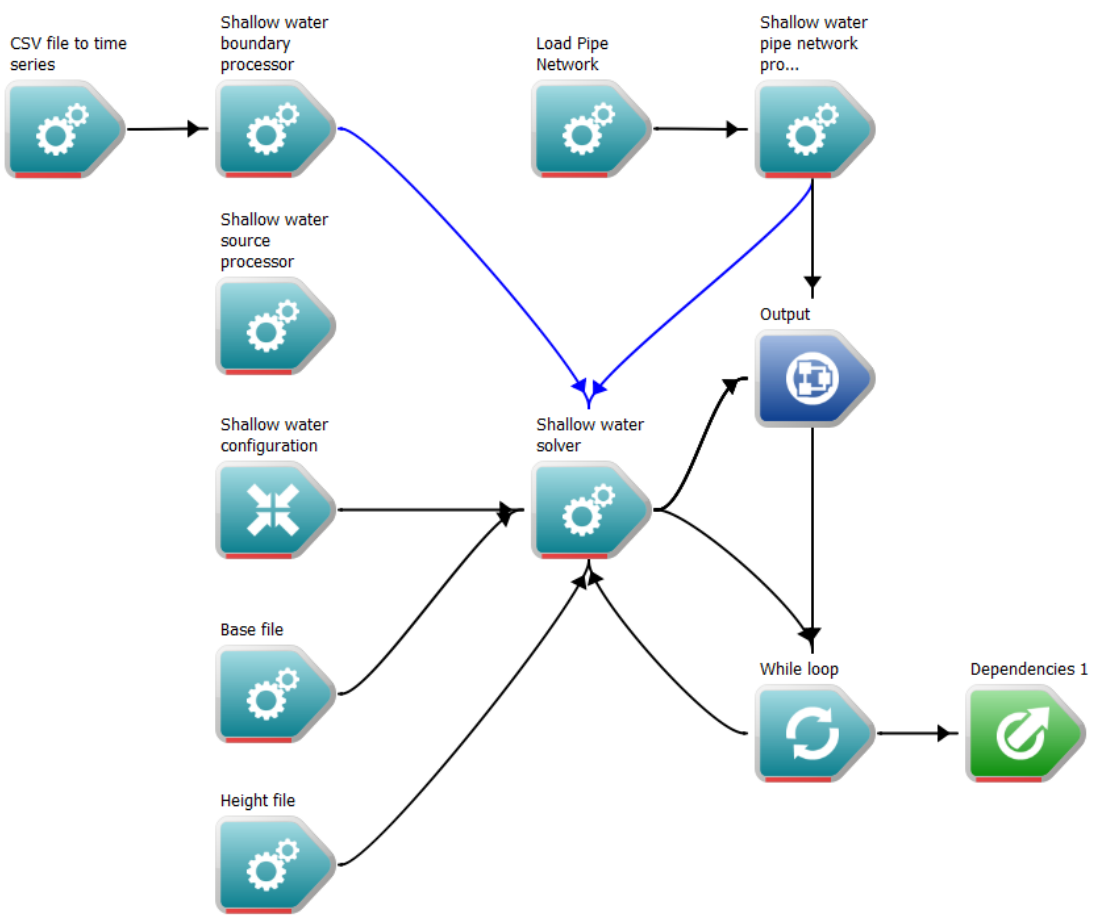

Fig. 3. Modelling components for the integrated mitigation modeling tool packaged using CSIRO Workspace: A scientific workflow platform
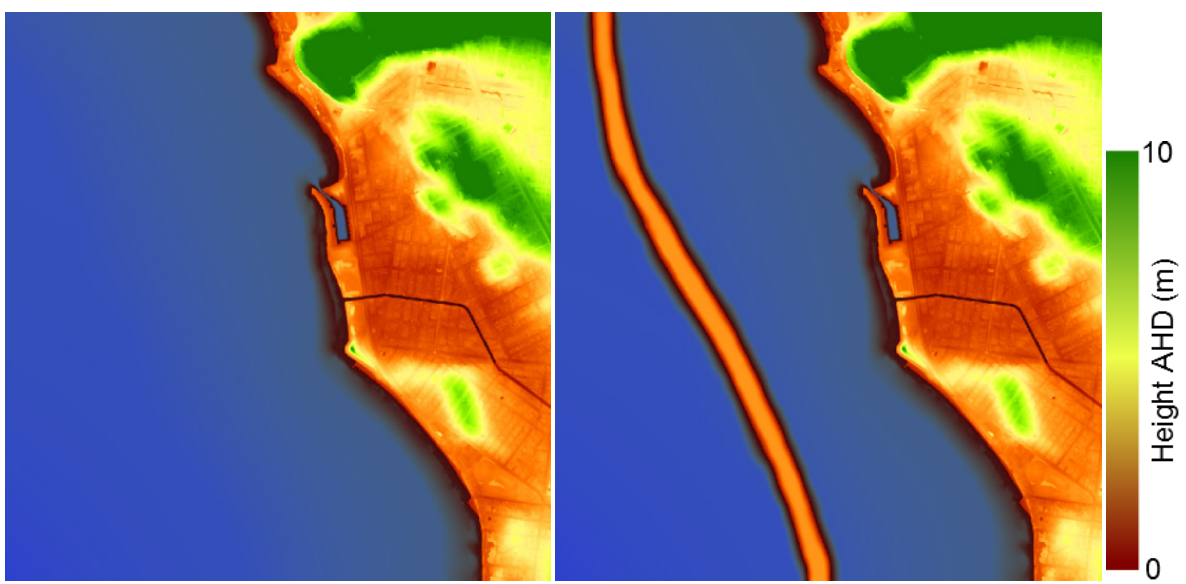

Fig. 4. The original topography and bathymetry (left) and modified with the addition of a $3 \mathrm{~m}$ off-shore reef (right). Initial water level shown in blue. 

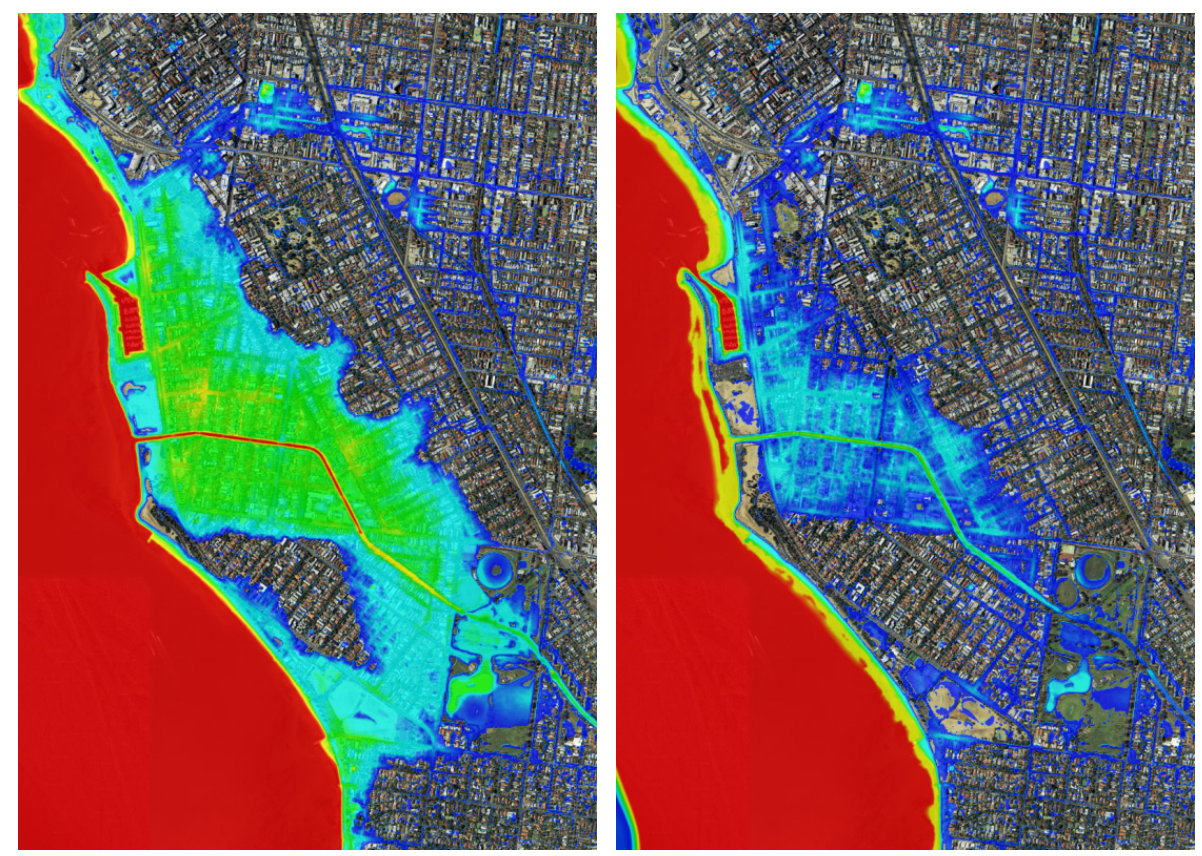

Fig. 5. Maximum flood extent with water coloured by depth without (left) and with (right) the off-shore reef for current day storm surge conditions

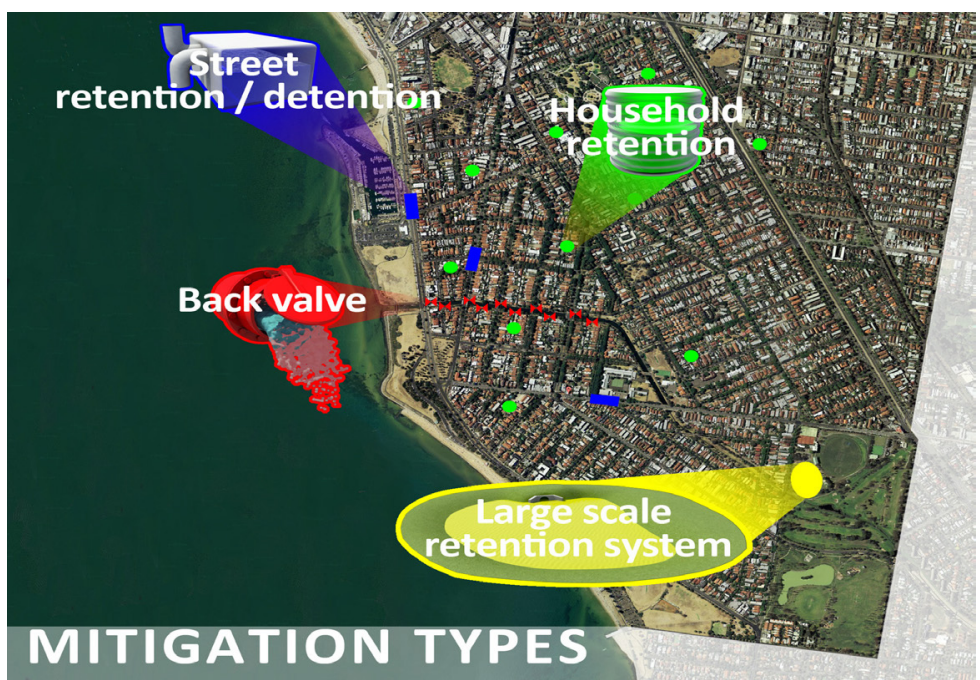

Fig. 6. Modular mitigation types and location around Elwood Canal 
The flood extent averaged over time for a $0.4 \mathrm{~m}$ sea level rise (SLR) scenario with and without mitigation is compared in Figure 7. This demonstrates that the mitigation options selected is able to reduce the flooding to essentially nuisance levels (retention times of only around $2 \mathrm{hrs}$ with flood height of $0.5 \mathrm{~m}$ or lower) in most regions around Elwood canal at a $0.4 \mathrm{~m} \mathrm{SLR}$.
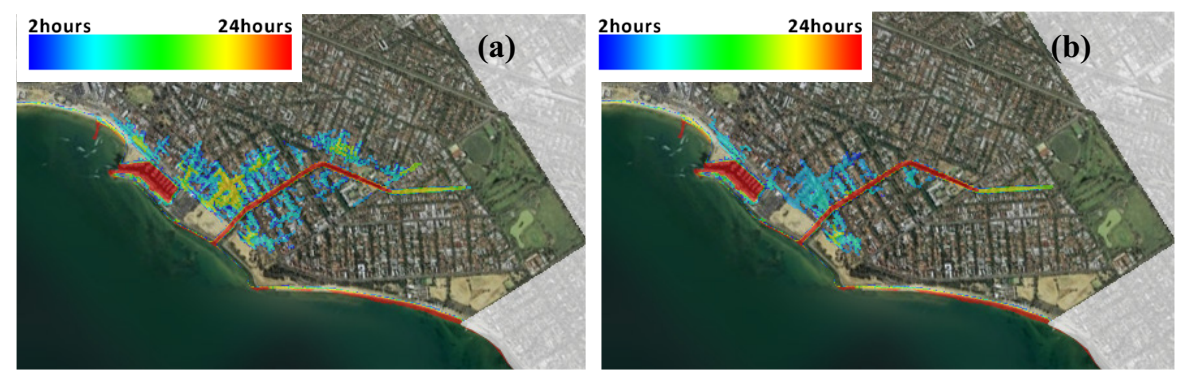

Fig. 7. Flood extent at $0.4 \mathrm{~m} \mathrm{SLR}$ (a) no mitigation (b) with mitigation. Water is coloured by retention time with blue indicating $2 \mathrm{hrs}$ and red $24 \mathrm{hrs}$.

\section{$8 \quad$ Advantages and Limitations}

a. Data inputs and methodology will necessarily change over time due to the nature of input data including LiDAR and climatic inputs. This model enables the capacity to feed in more updated datasets and methodology as they become available - this in turn provides a smarter and more cost-effective way to develop integrated flood simulations and test solutions. At present, no tool/model does this easily.

b. Usefulness of an integrated future flood modelling tool to land use and infrastructure managers - the capacity to gain a clear perspective about future integrated (coastal and catchment) flood behaviour provides enormous potential to understand the actual extent and risks associated with inundation over time. Current practice continues to model coastal and catchment flooding separately and creates a disjoint in providing this necessary perspective.

c. The capacity to simulate a range of coastal and drainage adaptation solutions into an integrated future flood model enables a range of testing and analysis of adaptation and drainage augmentation to measure what combination of solutions works, for what types of flooding, and for how long. This type of assessment capacity also provides a sound evidence base for consequent infrastructure design and an evidence-based cost benefit analyses of local and regional solutions.

d. Such a model also enables the capacity to assess and determine areas that will not be able to provide effective adaptation solutions - this then enables an evidence-base for the consideration of different land uses or drastic changes to current land use. 
e. The tool currently does not have the capacity to process point cloud LiDAR data and requires a "clean and processed" LiDAR input. CSIRO is aware of this limitation and work is underway to address this issue.

d. Visualisation of the results currently requires the LiDAR obtained terrain/bathymetry to match up with textural information. This is always not straightforward due to the inherent inconsistency associated with frames of reference for these layers. This issue is being investigated so that a Workspace based frame reference fixing algorithm can be provided to clients.

\section{Conclusions}

The flood modelling tool is an integrated system for stakeholders to investigate potential mitigation options in response to flood scenarios. The tool provides end-to-end modelling, analysis and visualization capability, as demonstrated by the City of Port Phillip case study. The model has also been developed to test the effectiveness of customised adaptation solutions. The next phase of development will be to customize the tool for use by land-use and infrastructure managers, especially in Local Government through a Port Phillip bay-wide approach.

Acknowledgements. The authors would like to acknowledge financial support provided by the City of Port Phillip and the CSIRO Digital Productivity and Services Flagship for conducting research towards developing the integrated flood mitigation analysis tool. The authors would also like to acknowledge the in-kind expertise and review provided by a range of key stakeholders including Melbourne Water, the Association of Bayside Municipalities, AECOM, and SGS Consulting to consider data and methodological inputs and likely adaptation solutions to be tested. Finally the authors would like to thank Fletcher Woolard from CSIRO for assistance in producing the visualisations related to this project.

\section{References}

1. AECOM, City of Port Phillip - Case Study for the Elwood Canal, Port Phillip Bay Coastal Adaptation Pathways Project (September 19, 2012).

2. Kurganov, A., Petrova, G.: A Second-Order Well-Balanced Positivity Preserving CentralUpwind Scheme for the Saint-Venant System, Commn. Math. Sci. 5, 133-160 (2007)

3. Neelz, S., Pender, G.: Benchmarking the latest generation of 2D hydraulic modeling packages, Report SC12000, Environmental Agency UK

4. Workspace, A.: scientific workflow platform, http://dx.doi.org/10.4225/ 08/544E4FF7B67B0 (accessed November 6, 2014)

5. Hoang, T.: Frequency analysis of tide levels for St Kilda marina (Site No: 229670) (Melbourne Water, 2011)

6. McInnes, K.L., O'Grady, J., Macadam, I.: The effect of climate change on extreme sea levels in Port Phillip Bay, Dept. of Sustainability and Environment, VIC report (2009) 